\title{
Investigation of the effect of moisture on the critical parameters of stability of frame-rod structural systems made of wood
}

\author{
Ksenia Dubrakova ${ }^{10000-0003-1951-7299]}$, and Victoriia Solodilova ${ }^{1 *[0000-0001-7287-1145]}$ \\ ${ }^{1}$ Southwest State University, 305040, 50 years of Octoberst. , Kursk, Russia
}

\begin{abstract}
The work of end moments and shear forces is taken as a criterion influencing the value of the limiting compressive force. It was found that the form of bifurcation of an individual element (active or passive) has a significant effect on the value of the critical parameters of stability of the entire system as a whole.

A frame-rod structural system made of wood is considered. The influence of humidity on the type of bifurcation of its compressed elements (constrained or forced) and the critical parameters of stability of the system as a whole under various modes of force loading are determined. It is shown that with a change in humidity, individual elements can go from passive loss of stability to active, which will change the value of the critical force by an average of $20 \%$.

To check the obtained analytical dependencies, experimental tests of frame-bar structures made of wood were carried out under their power static loading and variable humidity. At the same time, it was noted that the results of numerical and laboratory studies showed satisfactory agreement, on average by $95 \%$, which indicates the reliability of the developed methodology.
\end{abstract}

Keywords: stability, frame-rod system, active bifurcation, passive bifurcation, environmental loading, humidity.

\section{Introduction}

During the operation of structures, there is a change in the values of loads acting on structural elements, as well as a change in the size of sections, a change in natural factors, etc. For structures with supporting elements made of wood, the time of application of the load and the change in the humidity of the environment have a significant effect. In this regard, it is necessary to investigate the deformation of the rods with a change in the noted factors in conditions of constrained bifurcation and to establish the influence of these parameters on the critical parameters of the system.

An important issue in solving problems of stability of structural systems - the identification of the most dangerous elements or parts of a structure with low resistance to buckling was considered by AV Aleksandrov and V. Travush [1, 2]. Analysis of the influence of the modulus value of deformation and the rheological properties of wood on

*Corresponding author: bbty@yandex.ru. 
the reliability of structures is presented in the works of DK Arleninov [3, 4]. Stability problems for thin elastic plates compressed in one and two directions by uniformly distributed loads are considered in $[5,6]$. The strength and deformation characteristics of joints of wooden elements on inclined metal rods (NMS) without the use of glue, obtained from the results of testing samples of joints and structures of natural sizes, are given by VI Linkov [7]. AA Pogoreltsev concluded that it is necessary to differentiate the elastic moduli depending on the grade or class of wood strength, as well as on the operating conditions [8]. A. A. Morechkov proposed ways to reduce or completely eliminate the negative impact of these factors at the design and manufacturing stage, which would significantly increase the structural safety of glued wood structures [9]. The analysis of the occurrence of shrinkage cracks in large-span glued-board structures based on comprehensive examinations of load-bearing glued wooden structures of buildings for various purposes, operated in various temperature and humidity conditions. An analysis of the structural behavior of wood elements subjected to axial compression or combined axial compression and bending, taking into account nonlinear increases in deformations due to geometric and physical nonlinearity, is presented by S. B. Turkovskiy [10].

A review of existing studies on the stability of wooden structures under various loading conditions has shown that the issues of bifurcation of rod elements as part of structural systems made of wood in conditions of high humidity have not been sufficiently studied to date, is given in $[11,12]$. Meanwhile, such studies are necessary not only to study the features of deformation of loaded elements of wooden structures, to solve traditional problems of safety of structural systems, but also to assess the residual resource and protect the operated structural systems from progressive collapse caused by loss of stability of rod structures. In this regard, the study of the deformation of elements in loaded and under conditions of increased humidity of rod structural systems made of wood is of scientific interest and practically an important research task.

\section{Materials and methods}

When solving problems of determining the stability of structural systems by the displacement method, taking for unknown angles of rotation and displacement of nodes $Z_{1}, Z_{2}, Z_{n}$, we obtain a homogeneous system of equations for unknown angles of rotation and displacement of nodes $Z_{1}, Z_{2}, Z_{n}$ :

$$
\begin{gathered}
\mathrm{r}_{11} \cdot Z_{1}+\mathrm{r}_{12} \cdot Z_{2}+\cdots+\mathrm{r}_{1 n} \cdot Z_{n}=0 \\
\mathrm{r}_{21} \cdot Z_{1}+\mathrm{r}_{22} \cdot Z_{2}+\cdots+\mathrm{r}_{2 n} \cdot Z_{n}=0 \\
\cdots \\
\mathrm{r}_{n 1} \cdot Z_{1}+\mathrm{r}_{n 2} \cdot Z_{2}+\cdots+\mathrm{r}_{n n} \cdot Z_{n}=0
\end{gathered},
$$

where $Z_{i}(i=1,2, \ldots, n)$ - unknown angular and linear displacements; $r_{i, j}(i j=1,2, \ldots, n)$ are the coefficients of the displacement method, which depend on the values of the longitudinal forces in the rods.

$$
v_{i}=l \cdot \sqrt{\frac{P_{i}}{B_{r e d}(w, t)}},(i=1,2,3 \ldots n)
$$

In this case, in the formula (2) $B_{\text {red }}(w, t)$ is the reduced stiffness of the bar section, variable in time $(\mathrm{t})$ and wood moisture $(\mathrm{w}) ; v_{i}$ is a dimensionless parameter that depends on the magnitude of the longitudinal force.

Using the above equations, the critical parameters and forms of loss of stability of the considered structural system are determined from the condition of equality of the 
determinant of system (1) to zeroDet $=0$ and subsequent determination of the element's free length coefficient.

To determine the critical compressive stresses in the elements of structural systems, it is necessary to determine the coefficient of the free length of the element: $\mu_{i}=\frac{\pi}{v_{i}}$.

It is also of interest to assess the influence of the time of load application and changes in the moisture content of wood on the nature of the bifurcation of the rods in the structural system [13]. It can be expected that, under the influence of force and environmental factors, individual structural rods can go from passive to active buckling and vice versa, i. e. the inequality $\mathrm{A}_{i}<0$ will change its sign. Let us denote the initial parameter of the secular equation at a given constant load value and a fixed value of wood moisture $(\mathrm{w}=12 \%)$ :

$$
v_{i_{0}}=l \cdot \sqrt{\frac{P}{B_{0}}}
$$

Then the critical parameter of the secular equation $\mathrm{v}_{\mathrm{i}_{c r}}$, at which a transition is possible, for example, from passive to active bifurcation:

$$
\mathrm{v}_{\mathrm{cr}}=\mathrm{l} \cdot \sqrt{\frac{\mathrm{P}}{\mathrm{B}_{\mathrm{red}}(\mathrm{w}, \mathrm{t})}}
$$

Taking into account that the geometric length of the element remains constant, using formulas $(3,4)$, we determine the values $\mathrm{v}_{\mathrm{i}_{c r}}$, at which the work changes sign, these values will determine the transition from active loss of form of buckling to passive and vice versa.

The value of the reduced, time-variable (t) and wood moisture (w) stiffness of the cross-section of the rod, at which a transition from passive to active bifurcation is possible, is defined as:

$$
B_{\text {red }}(w, t)=\frac{v_{i_{\mathrm{\kappa p}}}^{2}}{{\mathrm{v}_{\mathrm{i}}}^{2}} \cdot B_{0} .
$$

In this case, the reduced time variable at a given wood moisture content is the stiffness of the cross-section of the bar in accordance with the formula [15-18]:

$$
\mathrm{B}_{\text {red }}(\mathrm{w}, \mathrm{t})=\frac{2 \cdot \mathrm{I} \cdot \sqrt{6 \cdot\left(1-0.913 \cdot \mathrm{t}^{0.38}\right)^{2}+2.25}-1.5}{\left(1-0.913 \cdot \mathrm{t}^{0.38}\right)^{2} \cdot \varepsilon\left(\mathrm{t}_{0}\right) \cdot\left(1+\mathrm{b} \cdot \mathrm{t}^{0,21}\right)}
$$

where $\varepsilon\left(t_{0}, t\right)$ is the relative deformation at the time of observation $t_{0}$, set taking into account the effect of the material's age, its aging properties, the mode and duration of loading (for the frame under consideration, $\varepsilon\left(\mathrm{t}_{0}\right)=\frac{P}{E \cdot A} ; \mathrm{b}-$ parameter depending on the moisture content of the wood (w).

Consider a two-span wooden frame in which the central pillar is loaded with a concentrated force $\mathrm{P}_{\mathrm{кp}}$, and the outer pillars are loaded with forces $\alpha \mathrm{P}_{\mathrm{cr}}$ Let us define the type of rod bifurcation (constrained or forced) $[13,14]$. We will calculate the frame by a quasi-static method of displacement using a step-iterative procedure and algorithm. 


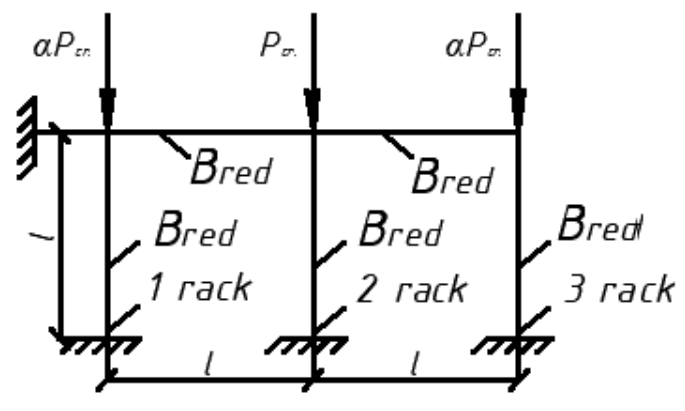

a)

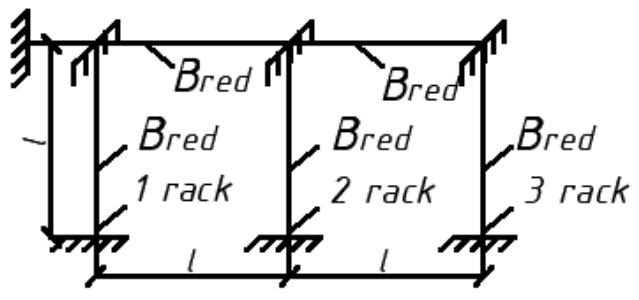

b)

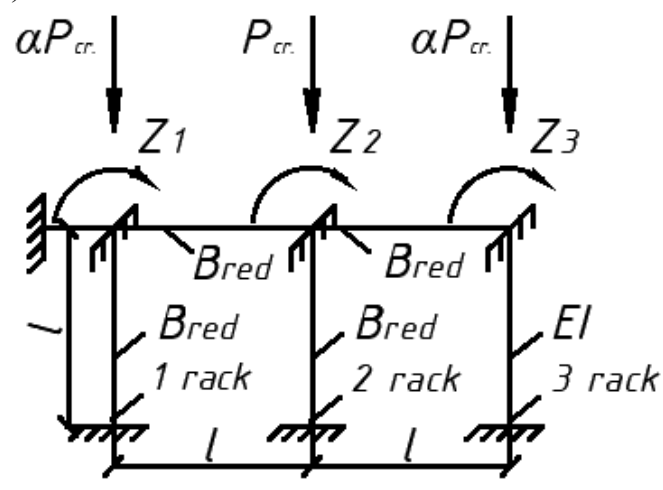

c)

Fig. 1. Design scheme of the frame (a), basic (b) and equivalent (c) systems of the method of displacement.

The determinant of system (1) for the frame under consideration after the corresponding reductions and transformations will take the form:

$$
4 \cdot \varphi_{2}^{3}\left(v_{1}\right)+20 \cdot \varphi_{2}^{2}\left(v_{1}\right)+34 \cdot \varphi_{2}\left(v_{1}\right)=0
$$

Having solved equation (7), we determine that the only real root is equal to $(-0,53)$, $\varphi_{2}\left(v_{1}\right)=-0,53$. The parameter of the secular equation of stability at the value of the load parameter $\alpha=1$ is determined from the table of functions of the displacement method for compressed-bent bars by interpolation: $v_{1}=5,04$. The value of the critical force is $\mathrm{P}_{c r} .=\frac{25,4 \cdot E \cdot I}{l^{2}}$. Therefore, the work of any frame post is equal to: 


$$
\mathrm{A}_{\mathrm{i}}=2 \cdot \mathrm{i} \cdot \varphi_{2, \mathrm{i}} \cdot \mathrm{Z}_{\mathrm{i}}
$$

In a similar way, an expression was obtained that discovers the determinant of system (1) at the value of the loading coefficient $\alpha=0,7\left(v_{1}=0,837, v_{2}=v_{3}\right)$. In this case, the coefficients are: $\varphi_{2}\left(v_{2}\right)=-1,2831, \varphi_{2}\left(v_{1,3}\right)=-0,1227$, which givefor frame struts 1 and 3 $v_{1,3}=4,65$, and $v_{2}=5,55$. The values of the critical forces for rack 2 are $\mathrm{P}_{c r, 2}=\frac{30,8 \cdot E \cdot I}{l^{2}}$, for racks 1 and $3 \mathrm{P}_{c r, 1,3}=\frac{21,63 \cdot E \cdot I}{l^{2}}$. The work of all frame racks is: $A_{1,3}=$ $0,245 \cdot i \cdot Z_{i}, A_{2}=-2,57 \cdot i \cdot Z_{i}$. Analyzing the operation of the frame struts, we can conclude that under normal operating conditions at the initial moment of loading time $(\mathrm{t}$ $=0$ day), the extreme frame struts lose stability actively, and the central frame passively.

With the value of the secular equation parameter $v_{1,3}=4,4$, the work of the frame racks 1,3 will change sign and become equal to $A_{1,3}=0,1296 \cdot i \cdot Z_{i}$, i. e. rods 1,3 will go to active bifurcationb [14].

The given example shows that when the stiffness of the struts changes, they can change the nature of buckling, i. e. go from active to passive and vice versa.

In addition, for posts 1 and 3 the free length is 0.6761 , and for post 2 it is 0.5661 . Consequently, the free length of frame struts should be determined taking into account the possibility of active and passive buckling [14].

For the frame shown in Figure 1 with the value of the load parameter $\alpha=0,7$ :

$$
\frac{2 \cdot \mathrm{I} \cdot \sqrt{6 \cdot\left(1-0.913 \cdot \mathrm{t}^{0.38}\right)^{2}+2.25}-1.5}{\left(1-0.913 \cdot \mathrm{t}^{0.38}\right)^{2} \cdot \varepsilon\left(\mathrm{t}_{0}\right) \cdot\left(1+\mathrm{b} \cdot \mathrm{t}^{0,21}\right)}=\frac{4,4^{2}}{4,65^{2}} \cdot B_{0}=0,8954 \cdot B_{0} \text {, }
$$

Having solved the equation (9), taking the humidity $\mathrm{w}=12 \%$, we determine the time $\mathrm{t}=$ 63 days, after which the rack of frame 1 will go to active bifurcation. We can say that after a time $t=63$ days, the critical force for the extreme frame strut (see Figs. 4, 2) will be equal to $\mathrm{P}_{c r, 1}=\frac{19,36 \cdot E \cdot I}{l^{2}}$.

Using criterion (9), for the frame under consideration, the change in the nature of the buckling of the struts was determined for various values of the load parameter $\alpha=0,5$; $\alpha=0,6 ; \alpha=0,7 ; \alpha=0,8 ; \alpha=0,9$ (Fig. 2) and with a change in the stiffness of the struts. The rigidity $B_{\text {red }}(w, t)$ was determined by the formula $(6)$. The results of this calculation are shown in Fig. 3. It can be seen from the figures that an increase in the loads on the extreme struts in time reduces the value of the critical force at which there is a loss of stability of the extreme struts [15]. The increase in wood moisture also has the same effect on the change in the critical force for the end posts.

Comparing the graphs $\langle\alpha-\mathrm{t}\rangle$ and $\langle\alpha-\mathrm{w}\rangle$ at a moisture content of $12 \%, 20 \%$ and $30 \%$ (curves 1, 2, 3, respectively), we can conclude that taking into account the moisture content of wood makes significant adjustments to the assessment of the stability of rod elements from wood and structural systems in general $[15,16]$.

Using criterion (6) for the frame under consideration, the change in the nature of the buckling of the struts was determined for different values of the load parameter $(\alpha=0.5$; $\alpha=0.6 ; \alpha=0.7 ; \alpha=0.8 ; \alpha=0.9$ ) in high humidity conditions [17]. The results of this calculation are presented in Figs. 2, 3. 


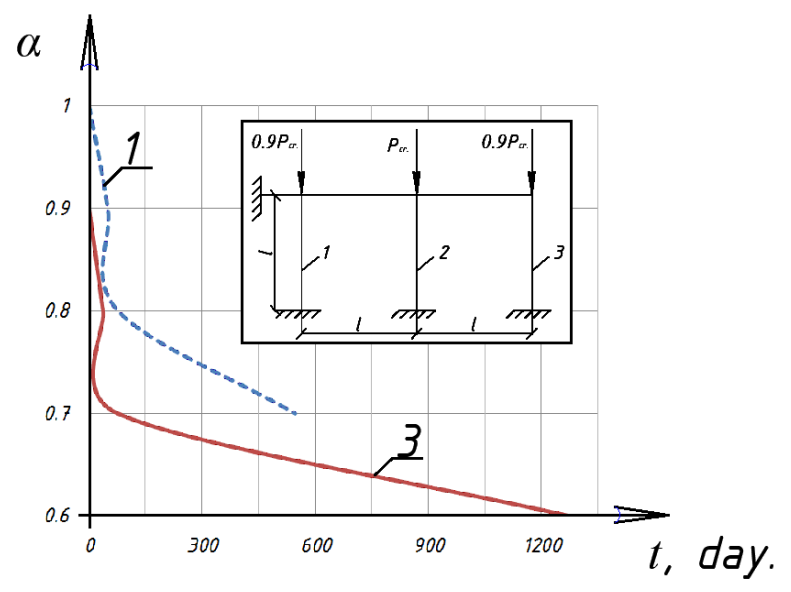

a)

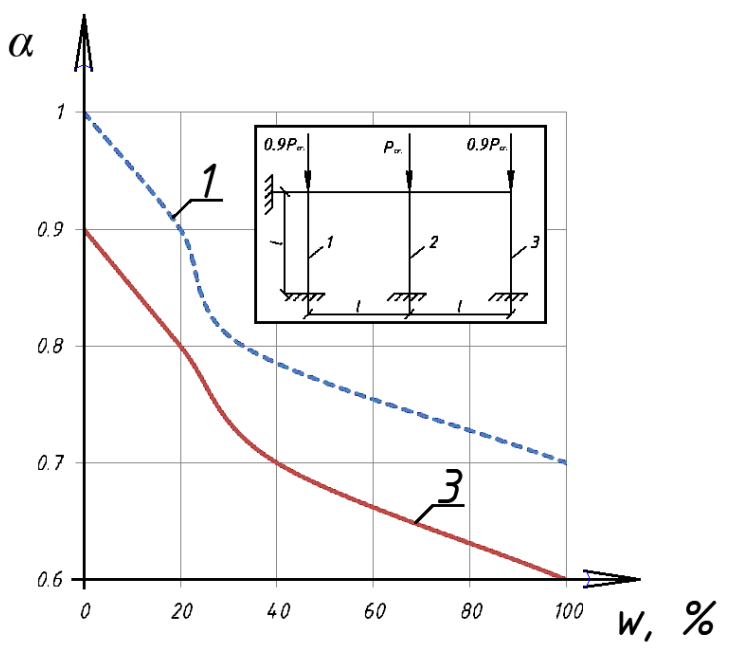

b)

Fig. 2. Changes in the nature of the buckling of the extreme struts $(1,3)$ at different values of the loading parameter $\alpha$ : a) from time to time; b) from humidity.

To analyze the above graphs, consider the change in the form of buckling of the frame struts in time at $\alpha=0.8$ (Fig. 3).

The mechanism of active and passive bifurcation in time can be clearly traced in Fig. $4 \mathrm{~b}$, from which it can be seen that when the frame is loaded in time from $t=0$ to $t=36$ days at $\alpha=0,8$ the middle strut experiences an "active» type of stability loss, and the extreme ones - passive, i. e. the case takes place when $A_{1}\left(M_{i}, Q_{i}\right)>0, A_{2}\left(M_{i}, Q_{i}\right)<0$, $A_{3}\left(M_{i}, Q_{i}\right)>0$. Within the time values from $\mathrm{t}=36$ days, until $\mathrm{t}=132$ days, the middle 2 and right 3 racks experience an active type of buckling, and the left rack 1 is passive, $i$. e. $A_{1}\left(M_{i}, Q_{i}\right)>0, A_{2}\left(M_{i}, Q_{i}\right)<0, A_{3}\left(M_{i}, Q_{i}\right)<0$. Whent $>132$ days, all frame struts undergo an active type of buckling, involving the entire structural system in a common bifurcation [18]. 


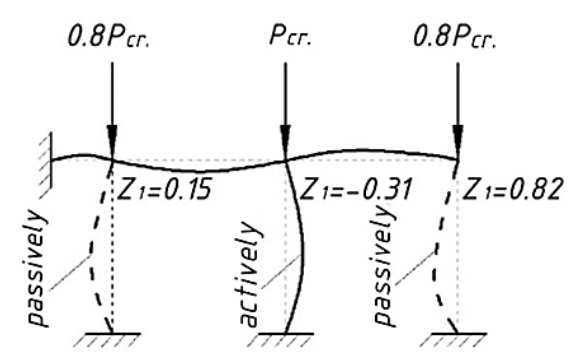

a)

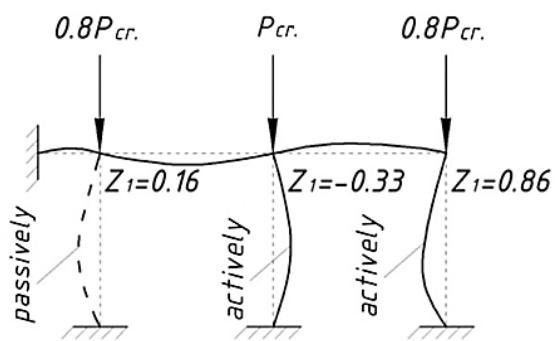

b)

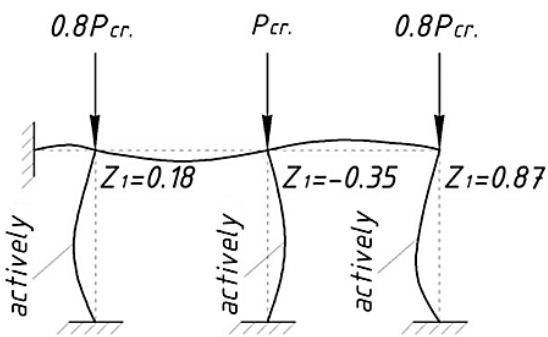

c)

Fig. 3 . Change in the form of loss of stability of the extreme frame struts with the parameter value $\alpha=0.8$ and humidity $12 \%$ in time: a) at $\mathrm{t}=0$ to $\mathrm{t}=36$ days; $\mathrm{b}$ ) from $\mathrm{t}=36$ days. up to $\mathrm{t}=132$ days; $\mathrm{c})$ at $\mathrm{t}>132$ days.

\section{Results and Discussion}

To check the obtained analytical dependencies in the laboratory of Southwest State University, six series of frame-bar structures made of wood were tested under their static loading and variable humidity (Figs. $4 \mathrm{a}, \mathrm{b}$ ).

In the course of the experiment, the deformations of the upper fibers were recorded using strain gauges installed on the surface of the frame strutsis presented by A. V. Matveev [19]. The deflection and change in the length of the bar during loading were measured with an electronic deflection meter, which made it possible to study the stability of compressed elements of a frame-and-bar structural system made of wood by increasing the load.

To create a simultaneous application of the load and environmental impact to the prototype, the experimental frames were placed in a sealed chamber before the experiment, in which the humidity was changed to a predetermined level. 


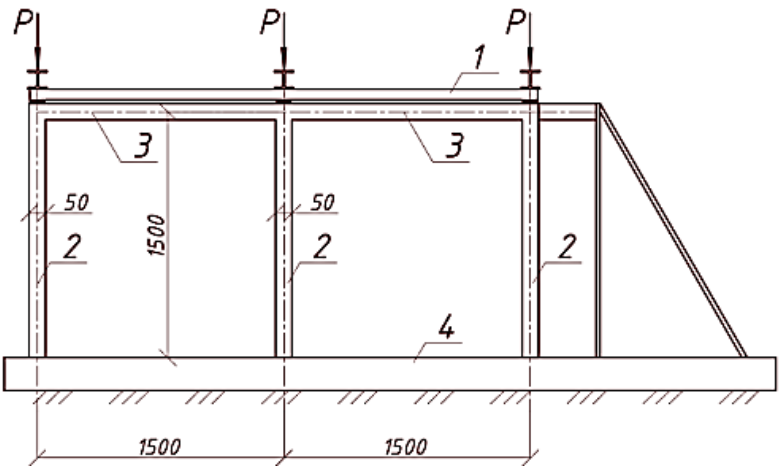

a)

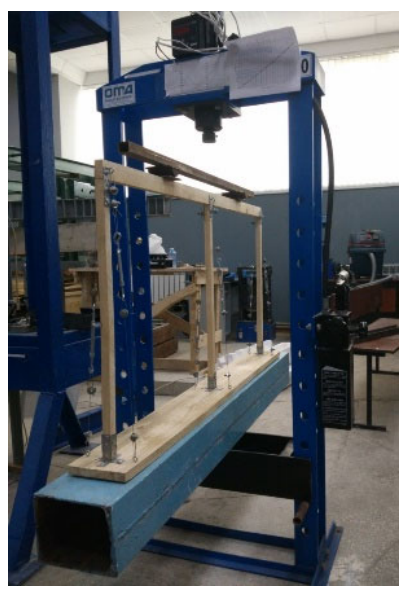

b)

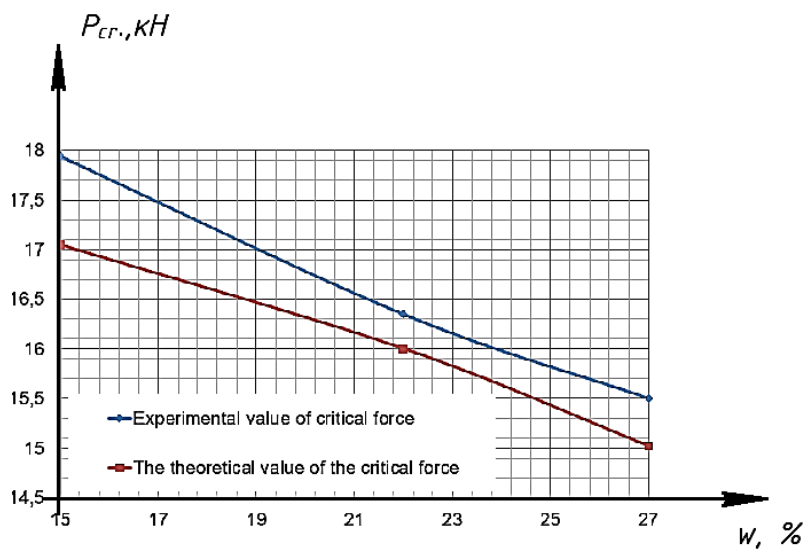

c)

Fig. 4. Scheme (a) and general view (b) of the installation and the results of assessing the stability of the frame-rod structural system made of wood (c): 1 - distribution beam; 2 - frame racks; 3 crossbar; 4 - support beam. 
A similar experiment helped to find out the numerical effect of moisture on the strength characteristics of wood. This result can be taken into account in new studies for the globalization of a material such as wood [20].

\section{Conclusion}

Environmental loads have a significant impact on the critical parameters of stability of frame-and-bar structural systems made of wood. With a change in humidity, individual elements can go from passive loss of stability to active, which will change the value of the critical force by an average of $20 \%$.

A method for calculating and experimentally testing the stability of frame-rod systems made of wood, taking into account environmental loads [20]. The results of numerical and laboratory studies showed satisfactory agreement, on average by $95 \%$, which indicates the reliability of the developed technique.

\section{Acknowledgements}

I'd like to express my gratitude to the rector of the Southwest State University Sergei GennadievichYemelyanov for the opportunity to engage in scientific activities. I'm grateful to my scientific advisor KseniaOlegovnaDubrakova for the invaluable experience passed on to me in the process of scientific research and sensitive mentoring.

\section{References}

1. A. V. Aleksandrov, V. I. Travush, A. V. Matveev, About the calculation of bar structures for stability, Industrial and civil construction, 3, 16-20 (2002)

2. A. V. Aleksandrov, V. I. Travush, A. V. Matveev, Investigation of the stability of the structures of the arched covering of the hall using the criteria for identifying the most dangerous elements, Russian Academy of Architecture and Construction Sciences RAASN, 8, 14-21 (2004)

3. D. K. Arleninov, About the new normative value of the elasticity modulus of wood. Industrial and civil construction, 3, 19-20(2013)

4. D. K. Arleninov, Estimation of the deflections of wooden beams during long-term loading . Industrial and civil construction, 11, 40-41 (2012)

5. M. G. Vanyushenkov, A. Y. Ushakov, Determination of the critical compressive load of elastic thin plates by the method of initial functions, Industrial and civil construction, 11, 71-73 (2010)

6. V. mI. Kolchunov, M. mV. Morgunov, L. V. Kozharinov, N. O. Prasolov, On the issue of algorithmization of the problem of calculating the survivability of reinforced concrete structures with loss of stability, Industrial and civil construction, 12, 77-79 (2012)

7. V. I. Linkov, Structures based on wooden elements of composite section with joints on inclined metal rods without the use of glue, Industrial and civil construction, 11, 29-31 (2012)

8. K. P. Pyatikrestovsky, A. A. Pogoreltsev, Substantiation of the normalized values of the elastic moduli in the calculations of wooden structures, Industrial and civil construction, 10, 33-35(2013) 
9. A. A. Smorchkov, S. A. Kereb, K. O. Baranovskaya, Safety of structures made of glued wood at the manufacturing stage, Industrial and civil construction, 12, 74-75 (2013)

10. S. B. Turkovsky, A. D. Lomakin, A. A. Pogoreltsev, Dependence of the state of glued wooden structures on the humidity of the surrounding air, Industrial and civil construction, 3, 30-32 (2012)

11. S. S. Gerhards, Bending creep and load duration of douglas - fir 2 by 4 s under constant load for up to 12-plus years, Wood and Fiber Science, 32(4), 489-501 (2000)

12. R. Hartnak, Langzeittragverhalten von druckbeanspruchtenBauteilenausHolz: dissertation, Weimar, 265 (2004)

13. S. G. Yemelyanov, E. G. Pakhomova, K. O. Dubrakova, S. V. Dubrakov, Stability of statically indefinite physically nonlinear tim-ber structural systems, Journal of Applied Engineering Science, 17, 3, 622, 404-407(2019) DOI: 10.5937/jaes1721686

14. S. G. Yemelyanov,E. G. Pakhomova, K. O. Dubrakova, Reliability of RC framebraced systems in dangerous geo-logical conditions, Journal of Applied Engineering Science, 17, 2, 602, 245-250 (2019) DOI: 10. 5937/jaes17-21685

15. V. I. Travush, V. I. Kolchunov, K. O. Dmitrieva, Long-term strength and stability of compressed wood rod, Construction and reconstruction, 5, 40-46 (2015)

16. V. I. Travush, V. I. Kolchunov, K. O. Dmitrieva, Stability of compressed wood rods with simultaneous manifestation of power and environmental impact, Building mechanics and calculation of structures, 2, 50-53 (2016)

17. V. I. Travush, V. I. Kolchunov, K. O. Dmitrieva, Experimental-theoretical study of the strength and stability of compressed wood rods under power and environmental impact, News of higher educational institutions. textile industry technology, 3, 280285 (2016)

18. K. O. Dubrakova, S. V. Dubrakov, F. V. Altuhov, D. H. Galaeva, The buckling of the physically nonlinear frame-rod structural systems, IOP Conf.: Mater. Sci. Eng., 698, 022007 (2019) DOI: 10.1088/1757-899X/698/2/022007

19. A. V. Matveev,Some issues of creating a specialized software package for the analysis of bridge structures, MIIT Bulletin, 7, 76-83 (2002)

20. K. O. Dubrakova, The stability issues of statically indefinable woodsystems,BST: Bulletin of construction equipment, 11(1011), 54-55 (2018) 